\title{
Biochemical, molecular and genetic aspects of fruit ripening in green-fruited and red-fruited tomato species
}

\author{
Slugina M.A. ${ }^{1 *}$, Shchennikova A.V. ${ }^{1}$, Dzhos E.A. ${ }^{2}$, Kochieva E.Z. ${ }^{1}$ \\ ${ }^{1}$ Institute of Bioengineering, Research Center of Biotechnology, RAS, Moscow, Russia \\ ${ }^{2}$ Federal Scientific Vegetable Center, VNIISSOK, Odintsovo region, Moscow district, Russia \\ *e-mail:mashinmail@mail.ru
}

Tomato fruit ripening is a complex process characterized by dramatic physiological and biochemical changes. It determines crop yield, and fruit color, flavor, dry matter content and shelf life. RIPENING INHIBITOR (RIN) is one of the main ripening adjusters controlling ethylene biosynthesis, cell-wall metabolism, sugar and secondary metabolite accumulation, and transcription factors expression. Wild and cultivated tomato species (Solanum sect. Lycopersicon) are known to be the most suitable model system to understand the mechanism of fleshy fruit ripening. The section Lycopersicon includes cultivated tomato $S$. lycopersicum and 12 wild relatives that differ significantly in fruit physiology. In this work, biochemical and expression analysis of red-fruited (RF) and green-fruited (GF) tomato species during ripening was performed. HPLC analysis showed that RF fruits accumulate glucose and fructose and change color from green to red as a result of carotenoid synthesis. Ripe fruits of GF tomatoes accumulate sucrose and are green due to the absence of carotenoids. The expression pattern of genes encoding RIN, sucrose hydrolyzing TAI (vacuolar invertase) and carotenoid-related PSY (phytoene synthase) was analyzed at four fruit developmental stages in GF and RF species. It was shown that the absence of sucrose degradation and carotenoid accumulation in GF species is due to the loss of TAI and PSY expression, at the same time the expression level of their activator RIN was high. Structural analysis revealed that in RF species, each of TAI and PSY promotors contain two RIN-binding sites, while in GF species, only one of two possible. Thus, the polymorphism of TAI and PSY promotor regions in RF and GF species may determine the differences in fruit morphophysiology.

Acknowledgements: The study was supported by Ministry of Science and Higher Education of the Russian Federation and Russian Science Foundation (No. 19-1600016). 